\title{
THE HOMOLOGY OF KUMMER MANIFOLDS
}

\author{
E. SPANIER
}

The theory of Kummer surfaces is a classical topic in algebraic geometry. ${ }^{1}$ A generalization to higher dimensions has been given by W. Wirtinger. ${ }^{2}$ The varieties introduced by Wirtinger may be called Kummer varieties and have algebraic dimension $n$ with $2^{2 n}$ ordinary double points. If these double points are desingularized in a standard manner by means of "dilatations," one obtains nonsingular varieties whose underlying manifolds will be called Kummer manifolds of complex dimension $n$ (topological dimension $2 n$ ).

The question of the torsion of Kummer varieties has been discussed recently by $A$. Andreotti ${ }^{3}$ since this author, however, did not desingularize the varieties under consideration, his results give no answer to the basic question whether they admit nonramified coverings or not. This question will be settled here by showing that the desingularized model mentioned above is simply connected; moreover, its homology groups will be determined for all dimensions. ${ }^{4}$

From a purely topological point of view all Kummer manifolds of a given dimension are homeomorphic to one another and may be defined as follows. Let $T$ denote the $2 n$-dimensional torus $(n \geqq 2)$ regarded as the $2 n$-fold product of the complex numbers of absolute value one with itself. For a subset $s$ of the integers $1, \cdots, 2 n$ let $p_{s}$ denote that point of $T$ defined by

$$
j \text { th coordinate of } p_{s}=\left\{\begin{aligned}
1 & \text { if } j \notin s, \\
-1 & \text { if } j \in s .
\end{aligned}\right.
$$

The points $p$, are the points equal to their inverses under the group structure on $T$. Let $G$ denote the group of transformations on $T$ consisting of the identity and the map $g: T \rightarrow T$ defined by $g(x)=$ the inverse of $x$ with respect to the group operation on $T$. For each $s$ let $B_{s}$ denote an open $2 n$-dimensional ball about $p_{s}$ with boundary $S_{s}$

Received by the editors April 19, 1955.

${ }^{1}$ Enzyk. d. Math. Wiss. III C 6 b (Castelnuovo-Enriques) no. 40.

2 Ueber eine Verallgemeinerung der Theorie der Kummerschen Fläche, Monatshefte für Mathematik und Physik vol. 1 (1890) pp. 113-128.

${ }^{3}$ Sopra il gruppo della torsione unidimensionale delle varietd algebriche, Rend. Sem. Mat. Torino vol. 12 (1952) pp. 239-264.

4 The author expresses his gratitude to Professors A. Weil and A. Borel, the former for suggesting the problem and the latter for helpful discussions about it. 
(a $(2 n-1)$-dimensional sphere) such that $G\left(B_{s}\right) \subset B_{s}$ and the map $g \mid S_{s}$ is the antipodal map. Let $\bar{X}=T-\cup B_{s}$; then $\bar{X}$ is a manifold with boundary $U S_{s} . G$ acts on $\bar{X}$ without fixed points, and we let $X=\bar{X} / G$ denote the orbit space of $\bar{X}$ under the action of $G$. Let $f: \bar{X} \rightarrow X$ denote the natural projection. $X$ is a manifold with boundary $U P_{\text {s }}$ where each $P$, is a real projective space of dimension $2 n-1$.

Consider the circle fibering $P \rightarrow M$ of $(2 n-1)$-dimensional real projective space over the complex projective space of complex dimension $n-1$ and let $C$ denote the mapping cylinder of this fibering. Then $C$ is a manifold with boundary $P$. For each $s$ let $C_{8}$ denote a copy of $C$ in which the boundary $P$ has been identified with $P_{8}$. Then $K=X \cup \cup C_{s}$ is the Kummer manifold of dimension $2 n$ (in the topological sense).

\section{THEOREM $1 . K$ is simply connected.}

Proof. We shall use $\Pi(Y)$ to denote the fundamental group of a space $Y$. Since each $B_{s}$ is a ball of dimension $2 n>2$, it follows that $\Pi(\bar{X}) \rightarrow \Pi(T)$ is an isomorphism onto; hence, $\Pi(\bar{X})$ is the free abelian group on $2 n$-generators $\bar{y}_{i}$ where $\bar{y}_{i}$ is represented by a path in $\bar{X}$ which is homotopic in $T$ to the $i$ th factor of $T$ considered as a closed path at $p_{s_{0}}$ where $s_{0}$ is the empty subset of $1, \cdots, 2 n$. A path representing $\bar{y}_{i}$ can be thought of as composed of four subpaths $a b_{i} c_{i}\left(g b_{i}\right)^{-1}$ where $b_{i}$ is a path in the $i$ th factor from a point $x_{0}$ on $S_{s_{0}}$ to a point $x_{i}$ on $S_{s_{i}}$ (where $s_{i}$ denotes the 1-element subset of $1, \cdots, 2 n$ consisting solely of $i$ ), $a$ is a path on $S_{\delta_{0}}$ from $g x_{0}$ to $x_{0}$, and $c_{i}$ is a path on $S_{s_{i}}$ from $x_{i}$ to $g x_{i}$. The element $g$ acting on $\Pi(\bar{X})$ sends each element into its negative. Since $\bar{X}$ is a double covering of $X$ with $g$ as covering transformation, it follows that $\Pi(X)$ is the extension of $\Pi(\bar{X})$ by an element of order 2 acting on $\Pi(\bar{X})$ to send every element into its inverse. Hence, if we let $y_{i}(i=1, \cdots, 2 n)$ denote the images in $\Pi(X)$ of the elements $\bar{y}_{i}$ of $\Pi(\bar{X})$ and let $z \in \Pi(X)$ denote the image of the nontrivial element of $\Pi\left(P_{s_{0}}\right)$ under the injection $\Pi\left(P_{s_{0}}\right) \rightarrow \Pi(X)$, we see that $\Pi(X)$ is the group generated by $y_{1}, \cdots, y_{2 n}$ and $z$ where the $y_{i}$ generate a free abelian subgroup and $z y_{i} z=y_{i}^{-1}$.

Under the natural map $f: \bar{X} \rightarrow X$ we see that $a$ maps into a representative $f a$ of $z$. Hence, $z y_{i}$ is represented by $f\left(b_{i}\right) f\left(c_{i}\right) f\left(\left[g b_{i}\right]^{-1}\right)$ $=f\left(b_{i}\right) f\left(c_{i}\right)\left[f\left(b_{i}\right)\right]^{-1}$. Now $f\left(c_{i}\right)$ represents a generator of $\Pi\left(P_{s_{i}}\right)$ so it follows that $\Pi(X)$ is generated by the images $\Pi\left(P_{s}\right) \rightarrow \Pi(X)$ (translated to a common base point).

From the exact sequence of homotopy groups

$$
\cdots \rightarrow \Pi_{2}(C) \rightarrow \Pi_{2}(C, P) \rightarrow \Pi(P) \rightarrow \Pi(C)=\Pi(M)=0,
$$


we see that $\Pi_{2}(C, P) \rightarrow \Pi(P)$ is onto (and also that $\Pi_{1}(C, P)=0$ ). Therefore, for each $s$, the map $\Pi_{2}\left(C_{s}, P_{s}\right) \rightarrow \Pi_{2}\left(P_{s}\right)$ is onto so by adding the $C_{s}$ to $X$ we kill all the elements of $\Pi(X)$ in the images of the $\Pi\left(P_{s}\right)$. Since we can add no new elements to $\Pi(X)$ by the addition of the $C_{s}$ (because $\left.\Pi_{1}\left(C_{s}, P_{s}\right)=0\right)$, it follows that $\Pi(K)=0$.

TheOREM 2. $K$ has no torsion and its Betti numbers $r_{i}$ are

$$
r_{i}=\left\{\begin{array}{l}
0 \text { if } i \text { is odd, } \\
1 \text { if } i=0,2 n, \\
\left(\begin{array}{c}
2 n \\
i
\end{array}\right)+2^{2 n} \text { if } i \text { is even and } 0<i<2 n .
\end{array}\right.
$$

Proof. We first obtain the cohomology structure of $\bar{X}$, then that of $X$ and finally that of $K$. We use three coefficient groups, namely, $R$ the reals, $J$ the integers, and $J_{2}$ the integers mod 2.

(a) The integral cohomology algebra $H^{*}(\bar{X} ; J)$ is an algebra generated by $u_{1}, \cdots, u_{2 n}$ of degree 1 and $v_{s}\left(s \neq s_{0}\right)$ of degree $2 n-1$ with the relations

$$
u_{i}^{2}=0, \quad u_{1} \cdots u_{2 n}=0, \quad v_{s} w=0(\text { any } w) .
$$

To prove (a) consider the exact triangle

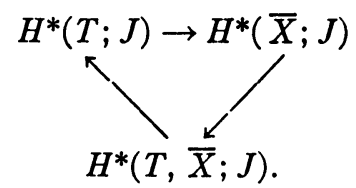

Now $H^{i}(T, \bar{X} ; J)=0$ if $i \neq 2 n$ and if $i=2 n$ is the direct sum of $2^{2 n}$ infinite cyclic groups. The map $H^{2 n}(T, \bar{X} ; J) \rightarrow H^{2 n}(T ; J)$ sends the $2^{2 n}$-tuple of integers $\left(n_{8}\right)$ into the integer $\sum_{8} n_{8}$. For each $s \neq s_{0}$ let $v_{s} \in H^{*}(\bar{X} ; J)$ be an element mapping into the $2^{2 n}$-tuple $\left(n(s)_{s^{\prime}}\right)$ where

$$
n(s)_{\boldsymbol{s}^{\prime}}=\left\{\begin{aligned}
0 & \text { if } s^{\prime} \neq s, s_{0}, \\
1 & \text { if } s^{\prime}=s, \\
-1 & \text { if } s^{\prime}=s_{0} .
\end{aligned}\right.
$$

Then the elements $\left(n(s)_{s^{\prime}}\right)$ generate the kernel of $H^{2 n}(T, \bar{X} ; J)$ $\rightarrow H^{2 n}(T ; J)$. The quotient of $H^{*}(T ; J)$ by the image of $H^{*}(T, \bar{X} ; J)$ is isomorphic to a subalgebra of $H^{*}(\bar{X} ; J)$, and the quotient of $H^{*}(\bar{X} ; J)$ by this subalgebra is isomorphic to the kernel of $H^{2 n}(T, \bar{X} ; J)$ $\rightarrow H^{2 n}(T ; J)$. Letting $u_{i}$ denote the image in $H^{1}(\bar{X} ; J)$ of the element of $H^{1}(T ; J)$ corresponding to the $i$ th factor of $T$, we see that the $u_{i}$ and $v_{0}$ for $s \neq s_{0}$ satisfy $(a)$. 
(b) We recall the following facts about the relation between the cohomology of $\bar{X}$ and that of $X$ which result from the fact that $\bar{X}$ is a double covering of $X^{5}{ }^{5}$

(1) Since $\bar{X}$ has no torsion, the only torsion coefficients of $X$ are 2 .

(2) The real cohomology algebra $H^{*}(X ; R)$ is isomorphic to the subalgebra of $H^{*}(\bar{X} ; R)$ invariant under $G$.

(3) The cohomology mod 2 is related by the exact sequence

$$
\cdots \rightarrow H^{i}\left(X ; J_{2}\right) \stackrel{f^{*}}{\rightarrow} H^{i}\left(\bar{X} ; J_{2}\right) \rightarrow H^{i}\left(X ; J_{2}\right) \rightarrow H^{i+1}\left(X ; J_{2}\right) \rightarrow \cdots
$$

Since the action of $g$ on $H^{*}(\bar{X} ; R)$ is given by $g\left(u_{i}\right)=-u_{i}$ and $g\left(v_{s}\right)=v_{s}$, it follows from (2) that the rational Betti numbers of $X$ are

$$
r_{i}(X ; R)=\left\{\begin{array}{l}
0 \text { if } i \text { is odd and }<2 n-1, \\
\left(\begin{array}{c}
2 n \\
i
\end{array}\right) \text { if } i \text { is even and }<2 n, \\
2^{2 n}-1 \text { if } i=2 n-1
\end{array}\right.
$$

To find the Betti numbers $\bmod 2, r_{i}\left(X ; J_{2}\right)$, note first that we have the exact sequence

$$
0 \rightarrow H^{0}\left(X ; J_{2}\right) \rightarrow H^{1}\left(X ; J_{2}\right) \stackrel{f^{*}}{\rightarrow} H^{1}\left(\bar{X} ; J_{2}\right) \rightarrow H^{1}\left(X ; J_{2}\right) .
$$

Therefore, $r_{1}\left(X ; J_{2}\right) \leqq r_{1}\left(\bar{X} ; J_{2}\right)+1=2 n+1$. On the other hand, $\Pi(X)$ was found during the proof of Theorem 1 , and from it we see that $H_{1}(X ; J)$ is the direct sum of $2 n+1$ groups of order 2 so $r_{1}\left(X ; J_{2}\right)$ $\geqq 2 n+1$. Hence, $r_{1}\left(X ; J_{2}\right)=2 n+1$ and $f^{*}: H^{1}\left(X ; J_{2}\right) \rightarrow H^{1}\left(\bar{X} ; J_{2}\right)$ is onto.

For $i<2 n-1, H^{i}\left(\bar{X} ; J_{2}\right)$ is generated by products of elements of $H^{1}\left(\bar{X} ; J_{2}\right)$ so we see that $f^{*}: H^{i}\left(X ; J_{2}\right) \rightarrow H^{i}\left(\bar{X} ; J_{2}\right)$ is onto for such $i$. It follows that in the exact sequence of (3) the map $H^{i}\left(\bar{X} ; J_{2}\right)$ $\rightarrow H^{i}\left(X ; J_{2}\right)$ is trivial for $i<2 n-1$ so $H^{i}\left(X ; J_{2}\right) \rightarrow H^{i+1}\left(X ; J_{2}\right)$ is an isomorphism into for $i<2 n-1$. Hence, for $i<2 n-1$,

$$
H^{i}\left(X ; J_{2}\right) \approx H^{i-1}\left(X ; J_{2}\right) \oplus H^{i}\left(\bar{X} ; J_{2}\right)
$$

whence

$$
r_{i}\left(X ; J_{2}\right)=\left(\begin{array}{c}
2 n \\
i
\end{array}\right)+r_{i-1}\left(X ; J_{2}\right)=\sum_{j \leqq i}\left(\begin{array}{c}
2 n \\
j
\end{array}\right) .
$$

6 These statements can be seen to follow from the results of S. D. Liao [A theorem on periodic transformations of homology spheres, Ann. of Math. vol. 56 (1952) pp. 6883]. (1) and (2) are easily verified directly from the fact that the cochain group of $X$ is isomorphic to the subgroup of the cochain group of $\bar{X}$ invariant under $g$. The sequence in (3) is the Gysin sequence of the fibering $\bar{X} \rightarrow X$ by 0 -spheres. 
At the top dimension we have the exact sequence $0 \rightarrow H^{2 n-2}\left(X ; J_{2}\right) \rightarrow H^{2 n-1}\left(X ; J_{2}\right) \rightarrow H^{2 n-1}\left(\bar{X} ; J_{2}\right) \rightarrow H^{2 n-1}\left(X ; J_{2}\right) \rightarrow 0$. Hence, $r_{2 n-1}\left(X ; J_{2}\right)+r_{2 n-1}\left(X ; J_{2}\right)=r_{2 n-2}\left(X ; J_{2}\right)+r_{2 n-1}\left(\bar{X} ; J_{2}\right)$ or

$$
\begin{aligned}
2 r_{2 n-1}\left(X ; J_{2}\right) & =\sum_{j \geqq 2 n-2}\left(\begin{array}{c}
2 n \\
j
\end{array}\right)+2 n+2^{2 n}-1 \\
& =2\left(2^{2 n}-1\right)
\end{aligned}
$$

so

$$
r_{2 n-1}\left(X ; J_{2}\right)=2^{2 n}-1=\sum_{j \leqq 2 n-1}\left(\begin{array}{c}
2 n \\
j
\end{array}\right) .
$$

Having the rational and mod 2 Betti numbers and remembering that all torsion coefficients are 2 we obtain the following result for the integral cohomology of $X$.

$$
H^{i}(X ; J)=\left\{\begin{array}{l}
0 \text { if } i \text { is odd and }<2 n-1, \\
J^{k} \text { if } i=2 n-1, \\
J^{p} \oplus\left(J_{2}\right)^{q} \text { if } i \text { is even and }<2 n,
\end{array}\right.
$$

where

$$
k=2^{2 n}-1, \quad p=\left(\begin{array}{c}
2 n \\
i
\end{array}\right), \quad \text { and } \quad q=\sum_{0 \leqq j \leqq i-1}\left(\begin{array}{c}
2 n \\
j
\end{array}\right) .
$$

(c) To obtain the cohomology of $K$ we use the exact sequence

$$
\cdots \rightarrow H^{i}(K, X ; J) \rightarrow H^{i}(K ; J) \rightarrow H^{i}(X ; J) \rightarrow H^{i+1}(K, X ; J) \rightarrow \cdots \text {. }
$$

Now $H^{i}(K, X ; J)$ is isomorphic to the direct sum of $H^{i}(C, P ; J)$ with itself $2^{2 n}$ times. It is easily verified that

$$
H^{i}(C, P ; J)=\left\{\begin{array}{l}
0 \text { if } i \text { is odd, } \\
J \text { if } i \text { is even and } \leqq 2 n
\end{array}\right.
$$

Hence, we see from the above exact sequence that $H^{i}(K ; J)=0$ if $i$ is odd and $<2 n-1$. Also $H^{2 n-1}(K ; J)$ is isomorphic to a subgroup of $H^{2 n-1}(X ; J)$; the latter is free so $H^{2 n-1}(K ; J)$ has no torsion. Since by duality the rank of $H^{2 n-1}(K ; J)$ equals the rank of $H^{1}(K ; J)$, which is zero, we see that $H^{2 n-1}(K ; J)=0$. Hence, the only nontrivial cohomology groups of $K$ are even-dimensional. From the duality theorem it follows that $K$ has no torsion. To complete the proof of Theorem 2 we need only calculate the ranks of the even dimensional cohomology groups of $K$. Using the exact sequence, it is easily seen 
that the rank of $H^{i}(K ; J)$ is the sum of the ranks of $H^{i}(X ; J)$ and $H^{i}(K, X ; J)$ so equals

$$
\left(\begin{array}{c}
2 n \\
i
\end{array}\right)+2^{2 n} \text { if } i \text { is even and } 0<i<2 n,
$$

and this completes the proof.

University of Chicago

\section{A FIXED POINT THEOREM FOR CONTINUOUS MULTI-VALUED TRANSFORMATIONS}

\section{ROBERT L. PLUNKETT}

1. Introduction. There are several different definitions of continuity for multi-valued transformations in existence in the literature [1]. Each definition is accompanied naturally by the question: which topological spaces $X$ have the property that, for each continuous multi-valued transformation $F$ of $X$ into $X$, there exists an $x \in X$ with $x \in F(x)$ ? This property is abbreviated F.p.p. and the point $x$ such that $x \in F(x)$ is called a fixed point under $F$. In [2], using one brand of continuity, Strother has shown that each closed and bounded interval $I$ of real numbers has the F.p.p., but that the square, $I \times I$, does not have it. Here, the concept of continuity will be the same as that in [2] and we shall answer the question above, restricting the topological spaces to be continuous curves (compact, locally connected, metric continua). More specifically, we shall prove that a continuous curve has the F.p.p. if and only if it is a dendrite [3, p. 88].

We shall employ the following characterization of continuity due to Strother [1]:

A multi-valued transformation $F$ of a space $X$ into a compact Hausdorff space $Y$ is continuous if and only if, for each $x_{0} \in X$, it is true that:

(1) $F\left(x_{0}\right)$ is closed,

(2) $V$ open and containing $F\left(x_{0}\right)$ implies that there exists an open set $U^{\prime}$ containing $x_{0}$ such that, if $x \in U^{\prime}$, then $F(x) \subset V$, and

Presented to the Society, April 16, 1955; received by the editors March 5, 1955. 